\title{
A NEW FILLING MATERIAL FOR COLD SLEEVE
}

The aim of this paper is to review material properties of a new filling material for cold sleeve. The first experimental measurements were performed on samples to determine required material properties. Then the structural analysis was performed for a pressurized pipe with insufficiently welded root and installed cold sleeve. The case of depressurized pipes that could cause a breach of cohesion between the filling material and surface of pipe or sleeve with the usage of cohesive finite elements was simulated.

Keywords: Repairing pipes, cold sleeve, stress of welded joint, cohesive finite elements

\section{Introduction}

Older metal pipelines have a lot of different types of material failures or defects [1]. Defects are identified during different actions on the pipelines, as are internal inspection methods, or other activities as making control probes, pipeline rehabilitation, searching gas-escape and similarly. Comparable carrying capacity of repair of the damaged pipe with the pipe without disturbance can be achieved by applying steel sleeves filled with composite epoxy [2]. Repairing the pipes with cold sleeve can reduce stresses at failure, and provide sufficient corrosion resistance of pipelines for the next operation. The disadvantages of these methods are low resistance and low axial tensions security protection in case of pressure medium seepage and short lifetime repairs. Installation of the proposed sleeve takes place in the full operation of the pipeline. The repaired segment of the pipeline is cleaned from the original coating. For maximum adhesion between a polymer filler and pipe surface or surface of the sleeve, these surfaces are cleaned. Subsequently, the two halves of the sleeve are mounted on the pipe and the space between the sleeve and the pipeline is defined by distance prisms. Then the sleeve is welded by the classical "V" weld and is sealed with a bandimex clamp and shrink wrap (Fig. 1). The tension spring creates space and conditions for a continuous, integral filling of the space between the sleeve and the repaired pipe. Finally, by using the filler the space between the sleeve and the pipe is filled by polymer. This type of sleeve is used for the repair of insufficiently welded roots too.

\section{Problem formulation}

For accurate reproduction of the stress state for all components of the cold sleeve, the procedure of cold sleeve installation has to be simulated. During the cold sleeve installation the pipeline is loaded by internal gas pressure and axial force. The cold sleeve and polymer adhesive are at stress-free state at this time. The cold sleeve and polymer are stressed when we change the value of internal pressure.

The polymer material used in the cold sleeve is based on PROTEGOL polymer. We note that PROTEGOL is polymer successfully used as anticorrosion protection on steel pipes and constructions placed under ground. It is one of the materials with the highest quality which is used for the rehabilitation of transit pipeline.

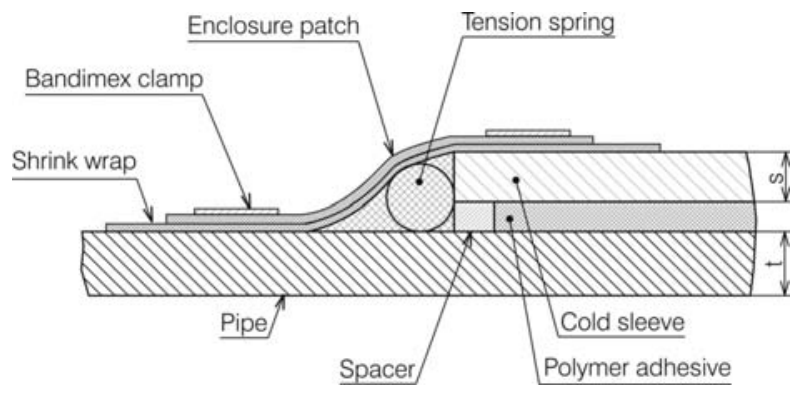

Fig. 1 Cross-section of installed cold sleeve

The size and shape of the weld is created in compliance with the norm STN 131075 (Slovak technical norm). In Fig. 1 is a cut through the pipe with a cold sleeve installed. For simulation the pipes with diameters 1220 and $1420 \mathrm{~mm}$ are considered.

The material properties of PROTEGOL based polymer were experimentally measured and the two-parameter Mooney-Rivlin hyperelastic constitutive model was used [3]. The quality of adhesion between the polymer layer and pipe or sleeve was characterised

\footnotetext{
* Pavol Novak, Milan Zmindak

Department of Applied Mechanics, Faculty of Mechanical Engineering, University of Zilina, E-mail: pavol.novak@fstroj.uniza.sk
} 
by the DPARAM parameter. The value of this parameter lies between 0 and 1 where 0 or 1 represents total adhesion or total separation of adjacent surfaces. Critical values of the DPARAM parameter were obtained when the pipe depressurisation occured.

\section{Experimental tests}

To get the material data needed to perform the finite element (FE) simulation, two experimental tests were made. The first of these was the tensile test of the modified polymer PROTEGOL. The tensile test was carried out in accordance with standard norm BS EN 10002-1. The results of this test are shown in Fig. 4 and they exhibit the statistical behavior of the specimens with a large variance of maximum force. The maximum force required to break the specimen was in the range $\langle 200,500\rangle \mathrm{N}$. The main reason for the large scatter of values is, in our opinion, the sensitivity of testing samples to humidity and temperature of the mixture during solidification. This affects the density and size of the bubbles contained



Fig. 2 Test sample

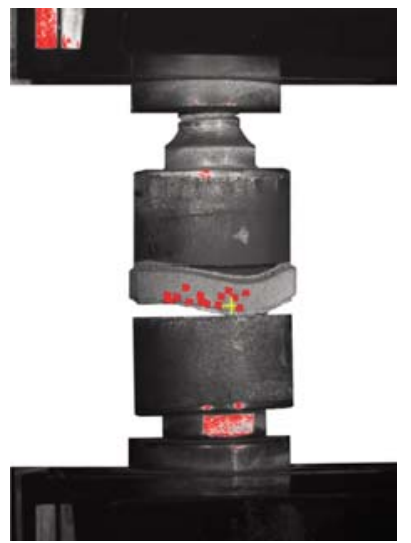

Fig. 3 Tearing of the test specimen in the sample. They act as stress concentrators. These factors are difficult to maintain the necessary limits in the application of technology in the exterior.

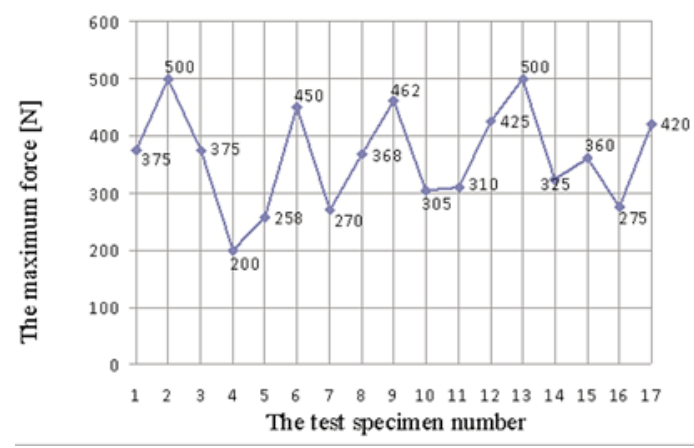

Fig. 4 Tensile test results
The next test was the tearing test. For this test cylindrical specimens were used (Fig. 2). The specimen was attached to the ZWICK tensile machine. Fig. 3 displays the tearing of the specimen in the tensile machine. The tearing test results showed the same behavior as the tensile test results. The maximal tearing force was $4150 \mathrm{~N}$ and minimal tearing force was $2153 \mathrm{~N}$ (Fig. 5).

\section{Finite element simulation}

Modeling with the finite element method was used to obtain stress state of all parts and risk assessment of debonding. The ANSYS Mechanical APDL was used for numerical simulation. The analysis was performed on the pressurized pipe with a subsequent depressurization to atmospheric pressure. The operating pressure was 7.35MPa. Based on the above given tests, we decided to use a two-parameter Mooney-Rivlin hyperelastic constitutive model for polymer. To determine the parameters of the Mooney-Rivlin model we broke 10 specimens. Additional three samples were used to tune the attachment to tensile testing machine and 4 specimens for optical tuning of the spray for the system ARAMIS [4]. From the performed FEM calculations we evaluated the separation of polymer from the surface of the pipe and the sleeve using the parameter $d_{n}$ or DPARM.

We used the axisymmetric FE model with additional plane symmetry. For the steel parts PLANE183 element was used [5]. This element has a quadratic displacement behavior. For the polymer part PLANE182 element was used. This element has a linear displacement behavior. The combination of PLANE183 element with the two-parameter Mooney-Rivlin model had convergence problems. Contact elements CONTA171 and TARGE169 with a cohesive zone material (CZM) model were used to simulate debonding of adjacent surfaces. The CZM model consists of a constitutive relation between the traction $T$ acting on the interface and the corresponding interfacial separation $\delta$ (displacement jump across the interface). We used the mode I dominated bilinear CZM model which assumes that the separation of the material interfaces is dominated by the displacement jump normal to the interface, as shown in Fig. 6 . The point A with coordinates $\left[\sigma_{\max }\right.$, $\bar{u}_{n}$ ] represents start of delamination and corresponds to DPARAM $=0$. The slope $\mathrm{Kn}$ is normal cohesive stiffness. The point $\mathrm{C}$ with



Fig. 5 Tearing force 
coordinates $\left[0, u_{n}^{c}\right]$ belongs to total separation of adjacent surfaces and corresponds to DPARAM $=1$. The partial delamination represents point B. In this case the $K_{n}\left(1-d_{n}\right)$ normal cohesive stiffness is used.

The simulation consists of two steps. In the first step only the pipeline under internal pressure was solved. The radial displacement was stored in the parameter and saved to disk. This parameter was used in a second step to modify geometry of the cold sleeve. This is necessary because the gap between the pipe and sleeve shall be defined in the pressurized pipeline. The second step consists of three substeps. In the first substep the complete model was solved (pipe with the installed cold sleeve) with internal pressure and axial force applied. In the second substep element kill/birth technique was used to ensure a stress-free state of the polymer filling and cold sleeve. In the last substep a depressurizing pipeline was simulated.

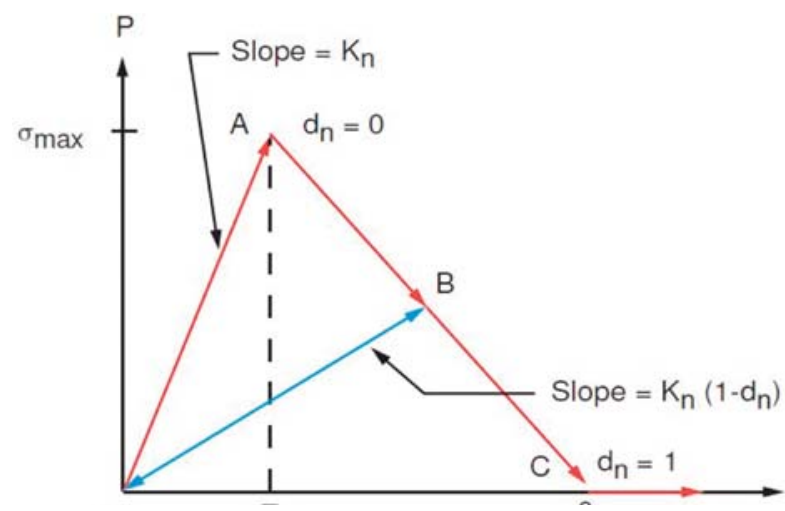

Fig. 6 Normal contact stress and curve of contact gap for bilinear mode I dominated CZM model

The simulation was executed for three geometric variants: variant 1 - pipe $\emptyset 1220 \mathrm{~mm}$, thickness $15.9 \mathrm{~mm}$, variant 2 - pipe $\emptyset 1220 \mathrm{~mm}$, thickness $13.5 \mathrm{~mm}$ and variant 3 - pipe $\emptyset 1420 \mathrm{~mm}$, thickness $15.6 \mathrm{~mm}$. For all three geometric variants the thickness of the cold sleeve was $12 \mathrm{~mm}$ and the thickness of a polymer layer was $8 \mathrm{~mm}$. To simulate the worst case scenario for debonding, material properties of the modified polymer were selected in this way: measurement with the highest stiffness for the Mooney-Rivlin model and measurement with the lowest tearing force for the CZM model $(2153 \mathrm{~N})$. The applied statically determined boundary conditions are described in Fig. 7. Gas pressure load is marked by red colour in Fig. 7.

Another load that needed to be considered was the axial load due to gas pressure in the closed pipe. This load is calculated as $F O=p . S$, where $S$ is a cross sectional area of the pipe. For the pipe with outer diameter $D=1220 \mathrm{~mm}$ and thickness $t=15.9 \mathrm{~mm}$ the resulting applied load is $F O=7.35 \times 3.14 \times 594.1 * * 2=$ 8.146.106 N. The pipe and sleeve are made from steel 11523 (S355J0). Elasticity modulus in tension is $E=206.0 \mathrm{GPa}$, Poisson's number is 0.30 and yield strength is $395 \mathrm{MPa}$. In Fig. 8 is a graph of engineering strain-stress for polymer PROTEGOL. The maximum strain is approx. $64 \%$ and maximum stress is approx. $4.5 \mathrm{MPa}$. The blue curve represents the measurement and pink curve represents the two-parameter Mooney-Rivlin approximation.

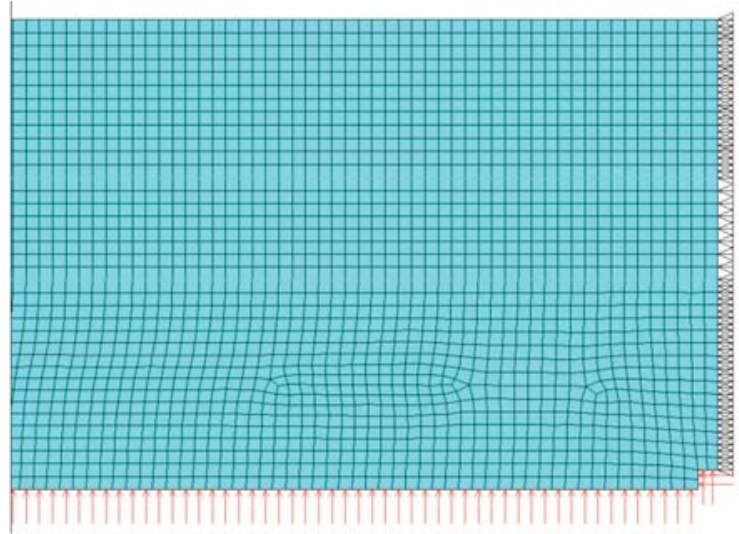

Fig. 7 Boundary conditions

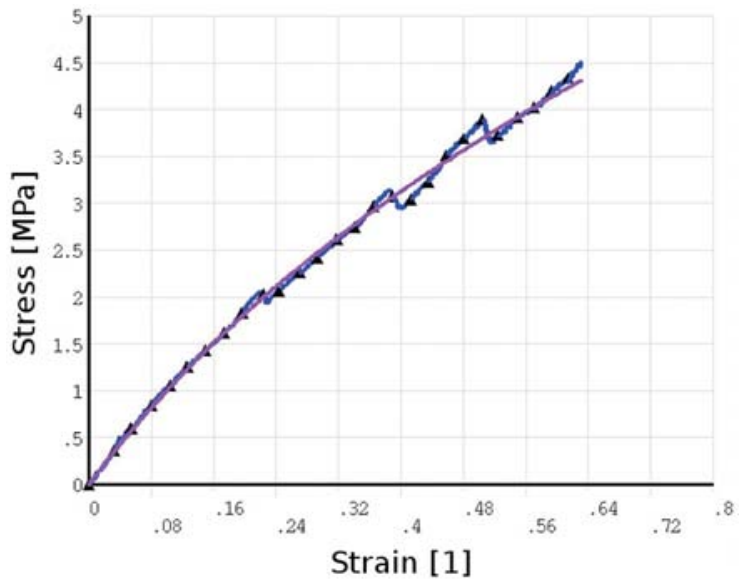

Fig. 8 Engineering stress - strain curves

The FEM calculation was performed as a geometric nonlinear analysis with elasto-plastic material properties of the pipe and sleeve [6].

\section{Analysis results}

In terms of the limit state of the load carrying capacity, vessels or piping are appreciated in terms of the primary stresses which are the results of acting a pressure in the piping. Table 1 summarizes the most important results of the analyses. It can be seen that the maximum value of the von Mises stress is $392 \mathrm{MPa}$. This value reaches almost the yield strength and occurs in the tip of the insuf- 
ficiently welded root. It is a singularity caused by a sharp corner, i.e. transition between the pipe and the insufficiently welded root. Fig. 9 shows the distribution of the contact gap between the polymer layer and the piping and the cold sleeve. The minus sign represents the separation of the adjacent surfaces. The cohesive failure is needed to reach the value of the contact gap $-2.5 \mathrm{~mm}$. Fig 10 . shows the detail of the contact gap at the cold sleeve beginning.

From Tab.1 it can be seen that the most critical variant of tearing is geometric variant 2 . The value of DPARAM $=0.669$ and contact gap is $-0.100 \mathrm{~mm}$.

Results in MPa for operating pressure $p=7.35 \mathrm{MPa}$

Tab. 1

\begin{tabular}{|c|c|c|c|c|}
\hline \multicolumn{2}{|c|}{} & Variant 1 & Variant 2 & Variant 3 \\
\hline \multicolumn{2}{|c|}{ Radial displacement $\left(u_{r}\right)[\mathrm{mm}]$} & -0.544 & -0.641 & -0.758 \\
\hline \multirow{2}{*}{$\begin{array}{c}\text { Radial stress } \\
\left(\sigma_{r}\right)\end{array}$} & Depressurized & 3.812 & 5.609 & 6.04 \\
\cline { 2 - 5 } & Pressurized piping & 90.826 & 112.037 & 107.729 \\
\hline $\begin{array}{c}\text { Circumferential } \\
\text { stress }\left(\sigma_{t}\right)\end{array}$ & Depressurized & -121.059 & -130.894 & -145.38 \\
\cline { 2 - 5 } & Pressurized piping & 347.888 & 414.005 & 412.931 \\
\hline $\begin{array}{c}\text { Axial stress } \\
\left(\sigma_{A}\right)\end{array}$ & Depressurized & -31.09 & -35.957 & -37.031 \\
\cline { 2 - 5 } & Pressurized piping & 282.299 & 339.009 & 339.089 \\
\hline \multirow{2}{*}{$\begin{array}{c}\text { Von Misses } \\
\text { stress }\left(\sigma_{A}\right)_{\max }\end{array}$} & Depressurized & 123.102 & 131.233 & 147.416 \\
\cline { 2 - 5 } & Pressurized piping & 329.108 & 391.191 & 391.627 \\
\hline \multicolumn{2}{|c|}{ Contact gap [mm] } & -0.060 & -0.100 & -0.086 \\
\hline \multicolumn{2}{|c|}{ D-param $[1]$} & 0.418 & 0.669 & 0.569 \\
\hline
\end{tabular}

Sensitivity analysis considering changes to the thickness of the polymer layer and thickness of the sleeve was performed. The thickness of the polymer layer was varied in the range of 4-8 $\mathrm{mm}$

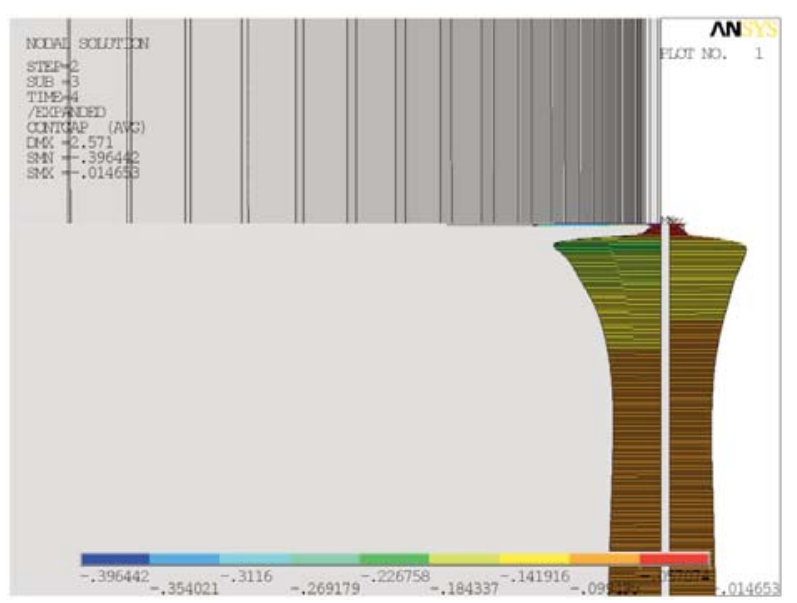

Fig. 9 Distribution of contact gap [mm] for variant 2, $p=7.35 \mathrm{MPa}$ in increments of $0.5 \mathrm{~mm}$. Fig. 11 shows the influence of the thickness of the polymer layer on the DPARAM parameter. This dependence is weak for a technologically useful range of the polymer layer thickness.

Other behavior of the DPARAM parameter is observed when we change the thickness of the sleeve. In this case, we carried out 15 variants with altered thickness calculation sleeve in the range of $5-12 \mathrm{~mm}$ in increments of $0.5 \mathrm{~mm}$. Fig. 12 shows the DPARAM parameter dependence on the sleeve thickness. This proportionality is very strong for the range of sleeve thickness from $9 \mathrm{~mm}$ to $12 \mathrm{~mm}$.

\section{Conclusion}

On the basis of the mentioned results we can conclude that repairing anomalous welds by means of the cold sleeve with a modified polymer PROTEGOL is safe with respect to a tearing polymer. With regard to the limit state, the pipeline, as well as the sleeves, are loaded in an elastic domain under the yield strength of the used steels. Since the problem was solved as a nonlinear problem with elastic-plastic behavior of materials, the results of the numerical simulation proved that plastic strains neither of the pipeline nor of the sleeve were reached.

\section{Acknowledgement}

The research work is partially supported by the Project of the Structural Funds of the EU, Operational Program Research and Development, Measure 2.2 Transfer of knowledge and technology from research and development into practice:

Title of the project: Development of Optimal Technology for the Analysis of the Limit States of Structural Elements in Contact, ITMS code: 26220220118

We support research activities in Slovakia/Project is cofunded from sources of ES.



Fig. 10 Detail of distribution of contact gap [mm] for variant 2, $p=$ 7.35 MPa considering axial force 


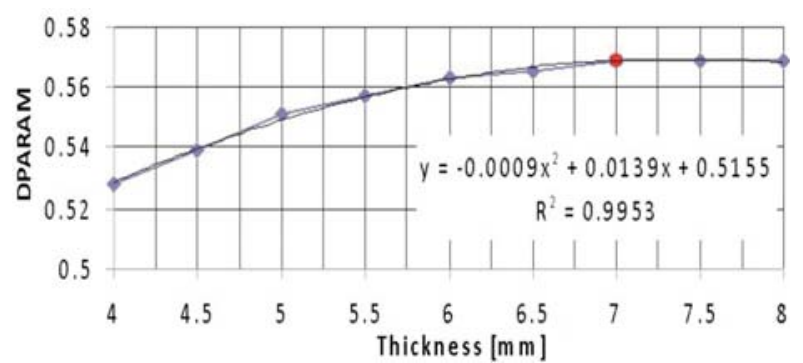

Fig. 11 Graph DPARAM vs. polymer thickness

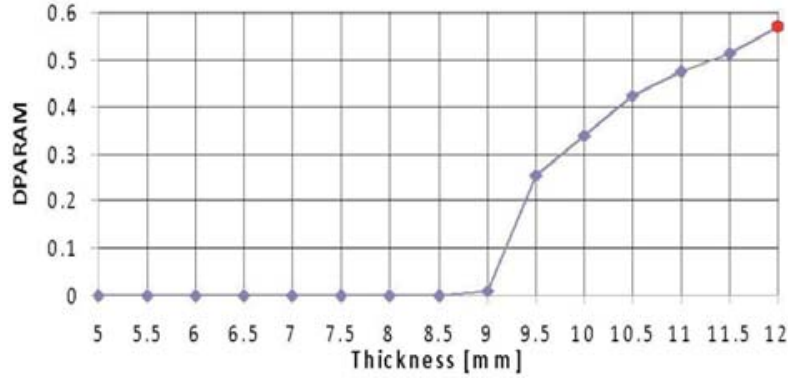

Fig. 12 Graph DPARAM vs. sleeve thickness

\section{References}

[1] KOPAS P., HANDRIK M., JAKUBOVICOVA L., SAGA, M.: Computational Simulations of Stress Distribution in Surrounding of Graphite Particles. Machine Dynamics Research, 2010, No. 3, Vol. 34, p. 115-123, ISSN 2080-9948.

[2] ZMINDAK, M., RIECKY, D.: Meshless modelling of laminate Mindlin plates under dynamic loads. Communications - Scientific Letters of the University of Zilina, 2012, vol. 14, No. 3, pp. 24-31, ISSN 1335-4205.

[3] ANSYS Release 11.0: Theory reference for ANSYS and ANSYS Workbench. ANSYS, Inc., 2007.

[4] ARAMIS - User information hardware, GOM MbH, www.gom.com.

[5] ANSYS Release 11.0: Element Reference. ANSYS, Inc., 2007.

[6] ANSYS Release 11.0: Structural Analysis Guide. ANSYS, Inc., 2007. 